e-Journal of Educational

Research, Assessment and Evaluation

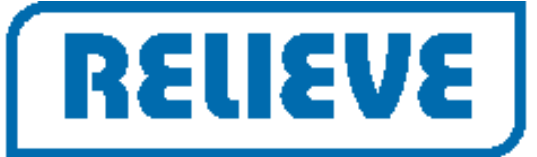

Revista ELectrónica de Investigación y EValuación Educativa

\title{
IMPACTO DE LA SOCIEDAD DEL CONOCIMIENTO EN LA UNIVERSIDAD Y EN LA COMUNICACIÓN CIENTÍFICA
}

\section{[Impact of the Knowledge Society in the University and in Scientific Communication]}

by/por

\begin{abstract}
Article record
About authors HTML format
\end{abstract}

\author{
Duart, Josep M. (jduart@uoc.edu) \\ Mengual-Andrés, Santiago (santiago.mengual@uv.es)
}

Ficha del artículo

$\underline{\text { Sobre los autores }}$ Formato HTML

\begin{abstract}
Over the last two decades as a result of the introduction and intensive use of technologies for information and knowledge in general, from the internet in particular, the university is living in a process of complete transformation that affects it's academic and organizational structures as well as the conception of educational methodology. The ICTs have demonstrated a need to establish coherent institutional strategies in their use and application along with the possibility to expand the sphere of institutional action in regards massive access to higher education. All of this shows an existing change from a model of education based on the transmission of knowledge, that was rather limited and under restricted access, to another that should fundamentally facilitate the competence to learn from people that live in the world in constant change, with open access to information and knowledge. In addition, all of this implies a transformation in the dynamics of communication and diffusion of scientific knowledge, that is now converted into something open and accessible which is subject to an analysis of knowledge social networks.
\end{abstract}

\section{Keywords}

Higher Education, Knowledge Society, e-learning, Open Acces, Open Science, Almetrics.

\begin{abstract}
Resumen
A la largo de las dos últimas décadas y como resultado de la introducción y uso intensivo de las tecnologías de la información y del conocimiento en general y de internet en particular, la universidad esta viviendo un proceso de transformación complejo que afecta a sus estructuras académicas y organizativas así como también a su concepción de metodología docente. Las TIC han evidenciado la necesidad de establecer estrategias de coherencia institucional en su uso y aplicación, así como la posibilidad de ampliar los ámbitos de acción institucional y el acceso masivo a la formación superior. Y todo ello pone de manifiesto el cambio existente de un modelo de formación basado en la transmisión del conocimiento, que estaba bien delimitado y bajo acceso restringido, a otro que debe fundamentarse en facilitar la competencia de aprender a personas que viven en un mundo en cambio constante y con acceso abierto a la información y al conocimiento. Todo ello, además, conlleva a una transformación en las dinámicas de comunicación y difusión del conocimiento científico, que se convierte en abierto, accesible y sometido al análisis de las redes sociales de conocimiento.
\end{abstract}

\section{Descriptores}

Educación superior, sociedad del conocimiento, elearning, acceso abierto, ciencia abierta, altmetric.
Tecnología, administración y pedagogía en la universidad: equilibrio y coherencia
La universidad como institución social ha experimentado importantes cambios durante las dos últimas décadas como resultado del 
impacto de la Sociedad de la Información conocida también como Sociedad del Conocimiento o Sociedad Red. Internet es base material y tecnológica de la Sociedad Red que ha generado nuevas formas de relación social que si bien no tienen su origen en internet no podrían haberse desarrollado sin su potencial (Castells, 2001). Estas nuevas formas de relación social han influido en todos los campos de la sociedad y, en especial, en las dinámicas de comunicación y de relación social.

Las dinámicas de enseñanza y aprendizaje han experimentado también cambios a partir de la introducción y uso intensivo de la tecnología y en particular, de internet. Aprender con uso intensivo de tecnología (e-learning) ha transformado las relaciones entre docencia $y$ discente, evidenciando la importancia del diseño y planificación en el proceso de aprendizaje (Cabero, 2006; Duart \& Repáraz, 2011).

Los cambios más sustantivos como resultado de la introducción y uso de internet en las dinámicas de las instituciones educativas se han sucedido en tres ámbitos: el del uso de la tecnología, el de la dinámica de la organización educativa, y el del paradigma educativo. En el ámbito de la tecnología hemos pasado en pocos años de la tecnología en el aula al aula en el bolsillo. Si bien en un inicio la introducción de la tecnología consistía en situar computadoras en el aula con programas educativos adaptados a las necesidades de los estudiantes actualmente, y gracias al desarrollo de los nuevos dispositivos móviles (smart phones, tablets, etc.) la realidad se sitúa en la portabilidad de la tecnología y su conectividad y sincronización (Ally \& Prieto-Blázquez, 2014). En el ámbito de la organización educativa hemos observado la aparición de nuevos perfiles profesionales, a menudo como resultado de procesos de adaptación de los perfiles profesionales ya existentes. Uno de los casos más evidentes es el de la transformación de las bibliotecas universitarias, antiguos repositorios de información estática, en centros de recursos para el aprendizaje y la investigación (CRAI) con documentación dinámica, accesible y global, con el cambio y la adaptación que comporta para sus profesionales (Connaway, 2014). También podemos destacar los cambios en la administración académica y la información y visibilidad de la actividad universitaria como resultado del uso de internet (Capshaw, 2012; McCaffery, 2010). En el ámbito educativo la introducción y uso intensivo de la tecnología ha resituado $\mathrm{y}$ redefinido el papel del docente y del estudiante, así como el de las fuentes de información y las metodologías para el aprendizaje (Bates \& Sangra, 2011).

La tecnología en general, e internet en particular, han sido motor de cambio en el mundo de la educación superior. Las transformaciones se han acontecido de forma rápida y a menudo con escasa planificación. De igual forma las innovaciones en tecnología son constantes y el incremento en la accesibilidad a la red ha ido incrementando de forma exponencial de tal forma que hemos modificado el concepto de brecha digital pasando de si se tiene o no acceso a la red a si se es competente o no en su uso (Castaño-Muñoz, 2010). De igual forma, los elementos básicos de control en la dinámica universitaria tradicional de costes, acceso y calidad que configuran el denominado Iron Triangle se ven trastocados por el uso de la tecnología. Los administradores educativos han procurado habitualmente maximizar el acceso y la calidad intentando controlar los costes. Un equilibrio complejo que la tecnología puede ayudar a mantener (Daniel, Kanwar, \& Uvalić-Trumbić, 2009).

Si bien los cambios y transformaciones más aparentes de las dos últimas décadas han sido los tecnológicos y los organizativos, en el ámbito universitario el reto continua siendo el de adoptar de forma coherente estos cambios con la finalidad de definir un modelo de aprendizaje transformador y adaptativo, adecuado para la comunidad educativa y la sociedad actual. El modelo de aprendizaje de la universidad de hoy debe responder a las expectativas y a las necesidades de las personas 
que formamos la sociedad actual, y de forma especial debe ofrecer herramientas que faciliten la construcción de competencias y capacidades adaptativas a las personas. Es un reto complejo que sólo puede lograrse transformando la institución actual y sus dinámicas educativas. Y para ello es necesario poner el foco de atención en el estudiante, en la persona que aprende. La coherencia entre el uso de una determinada tecnología, la adopción de una administración educativa y la metodología educativa empleada debe venir determinado por el perfil de la persona que va a aprender: el estudiante.

\section{Aprender en la sociedad red: “cómo” aprender y no "qué” aprender}

La relación formativa tradicional entre profesor y estudiante se ha modificado de forma substancial como resultado del uso intensivo de internet, afectando por igual a ambos actores del proceso educativo. Por una parte el estudiante quiere ser protagonista del proceso de aprendizaje, es decir, desea contar con los recursos necesarios para poder aprender e interactuar no sólo con el profesor si no con su comunidad de aprendizaje. Además prefiere gestionar y planificar su ritmo de estudio y seguir un modelo educativo personalizado con el apoyo continuado de profesores y/o expertos. El modelo educativo en la sociedad red se basa en los principios de interactividad, colaboración, personalización y flexibilidad (Sangrà, 2002). Y los retos educativos en la actualidad se sitúan en tres ámbitos en transformación: el de la hibridación, el feedback y la evaluación. La hibridación es el resultado del diseño formativo integrado de la formación presencial y de la no presencial o en línea. Los programas formativos híbridos permiten la creación de un continuo formativo real, propio de la dinámica de la formación continua o a lo largo de la vida (Osorio Gómez \& Duart, 2011a, 2011b). El feedback formativo es esencial en la dinámica educativa de la sociedad red y la interacción argumentada forma parte del proceso de enseñanza $y$ aprendizaje (Alvarez, Espasa, \& Guasch, 2012). Y los sistemas de evaluación integrada con el uso de la tecnología (e-assessment) son básicos para fundamentar las evidencias de los aprendizajes desarrollados en ambientes de aprendizaje complejos (Crisp, 2012).

El estudiante en la sociedad red está sometido a sobre-información como resultado del acceso fácil y continuado a la información existente en la red. De ahí que es necesario el desarrollo de competencias de selección y administración de la información. La formación continua y a lo largo de la vida es ya y será la pauta habitual de formación en una sociedad en constante cambio. De ahí que es fundamental entender la formación no únicamente como una sucesión de eventos o de hitos formativos en la vida sino como un continuo.

Es necesario poner el foco en el cómo aprender más que en el qué aprender (Cobo \& Moravec, 2011; Moravec, 2013). Los sistemas de enseñanza y de aprendizaje en la sociedad red necesitan superar la concepción de que educar es transmitir conocimiento para avanzar en la necesidad de preparar personas competentes para aprender. Este es sin duda el reto más importante con el que se encuentra la educación en la sociedad red. La introducción y el uso intensivo de la tecnología, e internet en particular, han modificado las relaciones y percepciones educativas y hacen evidente que ya no es posible basarse en transmitir un conocimiento que ya es accesible y que puede tener un elevado potencial de obsolescencia. Lo importante es tener la competencia de aprender a usar la red para acceder al conocimiento y usarlo de forma adecuada en nuestra actividad profesional.

\section{Internet en la universidad: nuevos modelos de educación superior}

La universidad actual, después de la incorporación y uso de internet en la dinámica educativa e institucional, debe sustentarse en un modelo educativo adaptativo e integrador que haga posible la adquisición y ejercicio de competencias sustantivas para la sociedad red actual. Para ello es necesario superar los marcos tradicionales, pero también aprender de ellos. 
No es necesario empezar de cero, existen ya experiencias significativas en el interior de muchas universidades y lo importante es saber canalizarlas y darles el empuje necesario para que puedan contribuir al cambio institucional (Bengoetxea, 2012; Weller, 2012).

Existen dinámicas transformadoras en el ámbito institucional de las universidades que están teniendo un gran impacto. Podemos mencionar cuatro de diferente índole pero con amplia relevancia: los recursos educativos en abierto (OER) y los cursos masivos (MOOC), el uso de datos para el análisis de comportamiento de estudiantes y profesores (Learning Analytics), y la globalización y multiculturalidad. El movimiento en abierto se inicia con los objetos de aprendizaje (Learning Objects) y la necesidad de encontrar un sistema estandarizado para poder compartir contenidos de aprendizaje (Downes, 2001). Posteriormente se lanzan los Open Educational Resources (Caswell, Henson, Jensen, \& Wiley, 2008) y universidades del mundo oocidental empiezan a abrir algunos de sus recursos educativos en la red. Se abre un debate sobre el business model existente detrás de ésta decisión, a la que sin duda ayudó también la aparición de la licencia de edición en abierto de Creative Commons. Y el siguiente paso ya son los cursos masivos en abierto o MOOC (Massive Open Online Courses) que plantean nuevas dinámicas educativas (Mengual-Andres, 2013; Roig, Mengual-Andres \& Suárez, 2014), de relación entre profesor, estudiante y comunidad, así como nuevos sistemas de evaluación (Martin, 2012), y aparecen estudios sobre si este movimiento en general y los MOOC en particular van a cambiar el modelo de universidad. (Anderson, Boyles, \& Rainie, 2012) Los sistemas de gestión del aprendizaje (Learning Management Systems) conocidos de forma genérica como Campus Virtuales ofrecen la posibilidad de recopilar y tratar ingentes cantidades de datos sobre el comportamiento de los miembros de la comunidad académica. De la minería de datos educativa se ha pasado al Learning Analytics en pleno momento de efervescencia del movimiento del Big Data. El análisis de los datos aporta información para valorar dinámicas de relación y establecer pautas de predicción de comportamientos para poder actuar sobre ellos, como es el caso del abandono en la universidad (Carnoy, Rabling, Castano-Munoz, Duart, \& Sancho-Vinuesa, 2011). En esta dinámica en abierto de interacción global se pone de manifiesto la necesidad de la construcción desde la universidad de redes globales de conocimiento. Estas redes y sus participantes muestran que la selección de los recursos o de los cursos se realiza teniendo en cuenta la reputación o prestigio del autor o del prescriptor, el rigor del contenido, y la flexibilidad del programa. De esta forma los new learners deciden su formación y completan su continuo formativo (Pedró, 2006).

En los nuevos modelos de educación superior los retos se sitúan en las dinámicas docentes y de aprendizaje. Si observamos la última edición del Horizon Report de Educación Superior (NMC, 2014) nos damos cuenta que los elementos de futuro más estratégicos corresponden a las prácticas docentes, de aprendizaje y a los sistemas de evaluación, mientras que ámbitos como infraestructura tecnológica y organización, que eran relevantes en informes anteriores, pierden fuerza ahora. Es el momento de focalizar en los métodos de aprendizaje, en cómo se aprende. Y las nuevas organizaciones educativas deberán dar respuesta a estas nuevas formas de aprendizaje. Sin embargo las estructuras académicas y organizativas de la universidad de hoy no facilitan el cambio. La universidad de mañana será diferente, y sobretodo, diversa. No existe ya un modelo único que replicar.

\section{La redefinición del papel de la universidad en la difusión del conocimiento y la ciencia abierta}

Podemos afirmar que el impacto de las TIC ha cambiado la velocidad de producción $\mathrm{y}$ distribución del conocimiento. Un ejemplo claro vendría dado por el reciente crecimiento en la publicación de documentos científicos derivados del e-journaling y los repositorios de 
ciencia de acceso abierto. En este sentido las nuevas tecnologías han desafiado las instituciones de Educación Superior de todo el mundo (Guri-Rosenblit, 2009), obligándolas a replantarse no solo sus modelos educativos, sino también su propósito como agentes en la difusión científica y su responsabilidad social.

Las universidades son organizaciones complejas, sus funciones inherentes las llevan a promover cambios significativos cuando existe una presión importante, aparecen nuevos modelos o cuando existe un colectivo de personas que demandan su participación (Toffler, 1985). Por ello las instituciones de educación superior se encuentran en la obligación (intrínseca en mayor medida) de contribuir y dar soporte a un sociedad en constante cambio, donde la tecnología es un agente evolutivo y transformador (Pittinsky, 2006) y la universidad debe posicionarse como la "torre conectada" que rompa la metáfora de la "torre de marfil" (De Pablos, 2010).

En suma, las entidades y centros de educación superior son importantes beneficiaros de las aportaciones de las TIC (Parker \& Burnie, 2009), dado que el impacto de ellas no solo son visibles en su gestión, los modelos educativos o sus políticas, sino también en otras áreas importantes de la vida universitaria, la publicaciones científicas (Guri-Rosenblit, 2009).

Desde una perspectiva económica global es observable que la deceleración económica que numerosos países están experimentando empieza a influir en la inversión tecnológica, describiéndose ciertos efectos de desregulación y falta de planificación (Anglada, 2014). Es del todo innegable cuestionar el papel de las universidades en la contribución científica y, por extensión, en la productividad que de la misma se desprende. La ciencia y la tecnología contribuyen al crecimiento de los estados de bienestar (Finch, 2012) produciendo beneficios económicos socialmente redistribuibles; por lo que se estima que una ciencia abierta genera más y mejor ciencia (Anglada, 2014). Por tanto no sorprende que estos beneficios en términos de productividad científica quieran -deban en parte- ser identificados, evaluados y medidos.

La medición de la productividad científica a día de hoy se centraliza en dos grandes índices: el Journal Citation Report (JCR) y el SCImago Journal \& Country Ranking (SJR). Estos listados permiten a instituciones, centros $\mathrm{o}$ estados no solo clasificar la importancia del impacto de sus agentes participantes sino, también, tomar decisiones respecto a las líneas de intervención en materia de política científica. Recientes informes han puesto de manifiesto la vinculación existente entre la productividad científica de un país (medida a través de su presencia en el JCR) y el crecimiento o desaceleración de su PIB. Las estrategias de las principales economías mundiales y en especial de los miembros del G20 se han puesto de manifiesto y vislumbran un panorama futuro de la regulación de la economía mundial (Thomson Reuters, 2014). El informe de Thomson Reuters ha manifestado la expansión en los últimos 10 años de la capacidad investigadora mundial así como la pérdida de competitividad de los líderes (hasta el momento) frente a las economías emergentes. El aumento del número de papers en WOS en un $145 \%$ por parte de Brasil, un $115 \%$ en India, un $373 \%$ en Arabia Saudi; el incremento en un $900 \%$ del número de patentes en China o el descenso del 3\% de la presencia europea en el JCR son algunos de estos indicadores de cambio (Thomson Reuters, 2014). Dichos datos no solo reflejan la relación entre desarrollo, ciencia e innovación, también describen las estrategias de determinados países (en especial las de aquellos con economías emergentes) respecto a las políticas científicas. Esta tendencia no es ni debe sorprendernos, la ciencia es un elemento fundamental para una sociedad industrializada, un propósito a alcanzar por los países considerados periféricos; es en suma una cuestión de supervivencia (Salager-Meyer, 2008). La triada ciencia, tecnología y publicación forma parte de la generación de riqueza, base de la verdadera independencia y de la gestación del equilibrio 
de deberes entre los países del mundo de hoy (Salager-Meyer, 2008).

En este contexto y mundo cada vez más competitivo son muchos los autores críticos con un sistema de medición que prioriza (en mayor medida) lo "mundial" a lo "local-periférico", y cuyos productos (elaborados por empresas) tan solo buscan la maximización de beneficios o maximización de audiencias. No es de extrañar también que números países (e.g: Japón) desarrollen iniciativas políticas destinadas a comercializar su ciencia académica, retrasando la publicación o publicando de forma incompleta para proteger el valor de sus hallazgos (patentes); situación que afecta adversamente a la ciencia abierta (Walsh \& Huang, 2014) y que incide significativamente sobre el bienestar social público. Este sistema está creando una serie de imposturas, desajustes y contradicciones que llevan a los investigadores $y$ grupos a cuestionar su participación en este engranaje de publicación (Gimenez Toledo, 2014), o de lo que realmente es, su papel en la contribución científica a la sociedad.

Lo anteriormente descrito -a modo de ejemplo- debe hacernos reflexionar sobre el verdadero papel de la universidad para con el mundo de la ciencia. Timothy Gowers, profesor de la Universidad de Cambridge, encabezó recientemente el movimiento "el coste del conocimiento" al qué rápidamente se unieron más de 10.000 investigadores de todo el mundo. Pero tal vez sea el proyecto del premio nobel Randy Schekman con la revista eLife el exponente más claro de una ciencia abierta, libre, de calidad, no comercializada y que se postula ajena a los índices de impacto "de la ciencia tradicional". Una revista concebida para los nuevos tiempos y que pone de manifiesto el negocio de las grandes editoriales y los problemas de los procesos de revisión (Schekman, Watt \& Weigel, 2013a). La paradoja de un proyecto científico de contribución social ha hecho que, sin pretenderlo ni solicitarlo, haya sido incluida en los listados JCR y WOS; no para beneficio de
eLife sino para el beneficio del resto de editores de dichos listados que ven como en sus artículos se incrementan el número de citas a papers de revistas de este calado. El caso eLife no es único, aislado o especial, Plos también ha cosechado el mismo éxito; ambos reflejo de iniciativas Open Acces (OA) comprometidas con una ciencia que contribuya a la sociedad de forma responsable. Cabe recordar que el factor de impacto nunca fue concebido para medir la calidad de los trabajos de investigación individual, sino como herramienta de comparación de revistas (Schekman, Watt \& Weigel, 2013b). Cabe por tanto reflexionar sobre los sistemas de medición, evaluación y clasificación que hemos construido alrededor de estos índices.

El movimiento OA aporta un valor extra para las instituciones (Aliaga, 2014). No solo está impulsando una ciencia de compromiso social, también contribuye a la mejora de la presencial internacional de la ciencia que se desarrolla en los países menos presentes en los índices selectivos internacionales (Alperín, Babini \& Fischman, 2014; Guo, Chue \& Li, 2014). Las estrategias de ciencia abierta para la divulgación del impacto del $\mathrm{I}+\mathrm{D}$ conllevan el desarrollo de la innovación empresarial, quedando demostrado el efecto positivo de la colaboración de las universidades en estos movimientos (Jong \& Slavova, 2014). Este discurso, más próximo a la responsabilidad de las universidades con el OA y su contribución social, es lo suficientemente importante para que las instituciones de educación superior se alejen del debate que quiere alimentarse respecto a los beneficios del OA en las métricas de citación (McCabe \& Snyder, 2014; Craig, Plume, McVeight, Pringle \& Amin, 2007).

Las TIC, los cambios sociales, las demandas de los ciudadanos, la necesidad de trabajar por una sociedad más igualitaria y el rol las instituciones de educación superior confeccionan un nuevo modo de entender la "ciencia 2.0" y, como tal, se presentan discusiones y estudios sobre los nuevos modos de establecer los factores de impacto de esta 
nueva ciencia (e.g: Hoffman, Lutz \& Meckel, 2014; Konkiel, Piwowar \& Priem, 2014) y comparaciones sobre su efectividad respecto a las formas tradicionales (Brown, 2014; Thelwall, Haustein, Larviere \& Sugimoto, 2013). Con todo, los debates sobre estos nuevos Article Level Metrics (ALM), formas emergentes de evaluación del impacto (o presencia) de la investigación derivadas de las posibilidades de la web social esta muy presente en la literatura científica internacional.

Establecer una comparación es una forma de abordar dicho debate, sin embargo también es posible ser más reflexivo: (a) presentar estos indicadores como nuevas formas de comunicación científica (Torres, Cabeza \& Jimenez, 2013), (b) proponer alternativas a la medición en base a la red social (Keita, 2012), (c) analizar el cambio de rol, técnicas y métodos de medición (Mehraban \& Mansourian, 2014) o (d) reconocer su complementariedad (Mounce, 2013) y el potencial de los ALM en los curriculum vite de los investigadores así como su papel equilibrador en la métrica tradicional (Piwowar \& Priem, 2013).

\section{Conclusión}

La universidad, como institución social, está en proceso de transformación. Sus estructuras académicas y organizativa se están adaptando a las nuevas dinámicas comunicativas, de acceso a la información y el conocimiento y de relación social. Pero la gran transformación es la que se deriva del cambio de paradigma en la metodología educativa, es decir, del paso de una cultura académica fundamentada en la transmisión del conocimiento a otra centrada en facilitar la competencia de aprender $y$ acompañar el proceso de aprendizaje. Las tecnologías de la información y del conocimiento, y en especial internet, están acelerando muchos procesos de transformación institucional debido a que facilitan el acceso a la información y al conocimiento. Pero las instituciones no pierden su razón de ser, se transforman $y$ adaptan dando respuesta a nuevas demandas competenciales y nuevos perfiles profesionales.

Sobre la comunicación científica y las nuevas fórmulas de medición que se ciernen sobre la publicación académica el debate no fácil. Asumir una postura $\mathrm{u}$ otra queda supeditado a la concepción que se tenga sobre la ciencia, sobre la forma de hacer ciencia y sobre el rol ciudadano que se adopte. Sin embargo no son únicamente las nuevas formas de medición del impacto lo que acontece, los beneficios del Open Data son indiscutibles y amplían los horizontes de la ciencia (Boulton, 2014). Este nuevo tipo de "hacer" ciencia permite disponer de datos en abierto, disponer del código en abierto, apoyar la replicación de los resultados, el refinamiento de teorías, leyes... y, es suma, ofrecer herramientas para una mejor educación (Light, Polley \& Börner, 2014). Los ALM suponen, sencillamente, una representación del impacto social de los trabajos académicos, un complemento e información representativa y necesaria.

Todo ello es, en suma, formas emergentes de entender $y$ hacer ciencia que empiezan a alejarse de los engranajes tradicionales. Es, finalmente, la intersección de una capa social y una capa tecnológica en una sociedad en red que demanda a las instituciones garantes de la ciencia un mayor compromiso social.

\section{Referencias}

Aliaga, F. M. (2014). Twenty years of electronic publishing and open access: a pioneer reaches maturity. RELIEVE, 20(1), art. 0. DOI: $10.7203 /$ relieve.20.1.3856

Ally, M., \& Prieto-Blázquez, J. (2014, January $15)$. What is the future of mobile learning in education?. RUSC. Universities and Knowledge Society Journal, 11(1), 142-151. DOI:10.7238/rusc.v11i1.2033

Alvarez, I., Espasa, A., \& Guasch, T. (2012). The value of feedback in improving collaborative writing assignments in an online learning environment. Studies in Higher Education, 37(4), 387-400. DOI: $\underline{10.1080 / 03075079.2010 .510182}$ 
Anderson, J., Boyles, J., \& Rainie, L. (2012). Higher Education: Experts Expect More Efficient Collaborative Environments and New Grading Schemes; They Worry about Massive Online Courses, the Shift Away. Retrieved from ERIC database (ED534048)

Anglada, L. (2014). De qué hablamos cuando hablamos de políticas de información. El Profesional de la Informacion, 23(2), 105112. DOI: $10.3145 /$ epi.2014.mar.01

Alperín, J. P. B., D.; Fischman, G. (2014). Open Access indicators and scholarly communications in Latin America. Ciudad Autónoma de Buenos Aires: UNESCO.

Bates, A. W. T., \& Sangra, A. (2011). Managing Technology in Higher Education: Strategies for Transforming Teaching and Learning. San Francisco: Willey.

Bengoetxea, E. (2012). Higher Education Governance Reform in Practice. Matching Institutional Implementation Practices and Policies. RUSC. Universities and Knowledge Society Journal, 9(29), 86-99. DOI: $\underline{10.7238 / \text { rusc.v9i2.1415 }}$

Boulton, P. G. (2014). The open data imperative. Insights, 27(2), 133-138. DOI: $\underline{10.1629 / 2048-7754.148}$

Brown, M. (2014). Is Almetrics an Acceptable Replacement for Citation Counts and the Impact Factor?. Serials Librarian, 67(1), 2730. DOI: 10.1080/0361526X.2014.915609

Cabero, J. (2006). Pedagogical bases of elearning. RUSC. Universities and Knowledge Society Journal, 3(1). DOI: 10.7238/rusc.v3i1.265

Capshaw, N. C. (2012). How the Internet Affects Higher Education: A Multi-Country Analysis. UK: AV Akademikerverlag

Carnoy, M., Rabling, B. J., Castano-Munoz, J., Duart, J. M., \& Sancho-Vinuesa, T. (2011). Who attends and completes virtual universities: the case of the Open University of Catalonia (UOC).Higher Education, 63, 53-82. DOI:10.1007/s10734-011-9424-0
Castaño-Muñoz, J. (2010, January 13). Digital Inequality Among University Students in Developed Countries and its Relation to Academic Performance. RUSC. Universities and Knowledge Society Journal, 7(1), 43-52. DOI: $10.7238 /$ rusc.v7i1.661

Castells, M. (2001). The Internet Galaxy: Reflections on the Internet, Business, and Society. New York: Oxford University Press. DOI: 10.1007/978-3-322-89613-1

Caswell, T., Henson, S., Jensen, M., \& Wiley, D. (2008, February 7). Open Content and Open Educational Resources: Enabling universal education. The International Review of Research in Open and Distance Learning, 9(1), 1-11

Cobo, C., \& Moravec, J. W. (2011). Aprendizaje invisible. Hacia una nueva ecología de la educación. Barcelona: UBe Universitat de Barcelona.

Connaway, L. S. (2014). ¿Cuál es el sentido de las bibliotecas? Un llamamiento para una evaluación centrada en el uso. BiD: textos universitaris de biblioteconomia $i$ documentació, 32. Retrieved from: http://bid.ub.edu/es/32/connaway2.htm\#stha sh.AkG1fivN.dpuf

Craig, I. D., Plume, A. M., McVeigh, M. E., Pringle, J., \& Amin, M. (2007). Do open access articles have greater citation impact?: A critical review of the literature. Journal of Informetrics, 1(3), 239-248. DOI: 10.1016/j.joi.2007.04.001

Crisp, G. T. (2012). Integrative assessment: reframing assessment practice for current and future learning. Assessment \& Evaluation in Higher Education, 37(1), 3343. DOI:10.1080/02602938.2010.494234

Daniel, J., Kanwar, A., \& Uvalić-Trumbić, S. (2009). Breaking Higher Education's Iron Triangle: Access, Cost, and Quality. Change: The Magazine of Higher Learning, 41(2), 30-35. DOI:10.3200/CHNG.41.2.30-35

De Pablos, J. (2010). Higher Education and the Knowledge Society. Information and Digital Competencies. RUSC. Universities 
And Knowledge Society Journal, 7(2), 6-15. DOI: $10.7238 /$ rusc.v7i2.977

Downes, S. (2001, July 1). Learning Objects: Resources For Distance Education Worldwide. The International Review of Research in Open and Distance Learning, 2(1). Retrieved from: http://www.irrodl.org/index.php/irrodl/articl e/view/32/378

Duart, J. M. \& Repáraz, C. (2011). Enseñar y aprender con las TIC. Estudios Sobre Educacion, 20, 9-19

Finch, J. (2012). Accessibility, sustainability, excellence: how to expand access to research publications. Report of the Working Group on Expanding Access to Published Research. Findings, 2012.

Gimenez Toledo, E. (2014). Imbalance in scientific publishing. Revista de Investigacion Educativa, 32(1), 13-23. DOI: $\underline{10.6018 / \text { rie.32.1.190251 }}$

Guo, F., Xue, J. Y., \& Li, R. X. (2014). Open access in China: A study of social science journals. Journal of Scholarly Publishing, 45(4), 336-352. DOI: 10.3138/jsp.45.4.02

Guri-Rosenblit, S. (2009). Digital Technologies in Higher Education. New York: Nova Science Publishers.

Hoffmann, C. P., Lutz, C., \& Meckel, M. (2014). Impact factor 2.0: Applying social network analysis to scientific impact assessment. Proceedings of the Annual Hawaii International Conference on System Sciences, 1576-1585.

Jong, S., \& Slavova, K. (2014). When publications lead to products: The open science conundrum in new product development. Research Policy, 43(4), 645654. DOI: 10.1016/j.respol.2013.12.009

Keita, B. (2012). Altmetrics: alternative ways of measuring scholarly impact based on the social web. Journal of Information Processing and Management, 55(9), 638646. DOI:10.1241/johokanri.55.638

Konkiel, S., Piwowar, H., \& Priem, J. (2014). The imperative for open altmetrics. Journal of Electronic Publishing, 17(3). DOI: $\underline{10.3998 / 3336451.0017 .301}$

Light, R. P., Polley, D. E., \& Borner, K. (2014). Open data and open code for big science of science studies. Scientometrics, 101(2), 1535-1551. DOI: $10.1007 / \mathrm{s} 11192-$ 014-1238-2

Martin, F. G. (2012). Will massive open online courses change how we teach? Communications of the ACM,55(8), 26. DOI: $10.1145 / 2240236.2240246$

McCabe, M. J., \& Snyder, C. M. (2014). Identifying the effect of Open Access on citations using a panel of science journals. Economic Inquiry, 52(4), 1284-1300. DOI: $\underline{10.1111 / \text { ecin.12064 }}$

McCaffery, P. (2010). The Higher Education Manager's Handbook: Effective Leadership and Management in Universities and Colleges. New York: Routledge

Mehraban, S., \& Mansourian, Y. (2014). Tracing scientific trends: Scientometrics methods and metrics, and the change in librarians' roles. Iranian Journal of Information Processing Management, 29(3), 613-631.

Mengua-Andrés, S. (2013). Rethinking the role of Higher Education. Journal of New Approaches in Educational Research, 2(1), 1-2. DOI: $10.7821 /$ naer.2.1.1-2

Moravec, J. W. (2013). Knowmad society: the "new" work and education. On the Horizon, 21(2), 79-83. DOI: $\underline{10.1108 / 10748121311322978}$

Mounce, R. (2013). Open Access and Altmetrics: Distinct but Complementary. Bulletin of the American Society for Information Science and Technology, 39(4), 26-29. DOI: 10.1002/bult.2013.1720390406

NMC. (2014). NMC Horizon Report > 2014 Higher Education Edition | The New Media Consortium. Retrieved November 12, 2014, from http://www.nmc.org/publications/2014 -horizon-report-higher-ed 
Osorio Gómez, L. A., \& Duart, J. M. (2011a). A hybrid approach to university subject learning activities. British Journal of Educational Technology, 43(2), 259-271. DOI: $10.1111 / \mathrm{j} .1467-8535.2011 .01175 . \mathrm{x}$

Osorio Gómez, L. A., \& Duart, J. M. (2011b). Interaction Analysis in Hybrid Learning Environment. Comunicar, 19(37), 65-72. DOI:10.3916/C37-2011-02-06

Parker, B., \& Burnie, D. (2009). Classroom Technology in Business Schools. AACE Journal, 17(1), 45-60.

Pedró, F. (2006). The New Millennium Learners: Challenging our Views on ICT and Learning. IDB Publications (Working Papers). Retrieved from http://ideas.repec.org/p/idb/brikps/9228.html

Pittinsky, M. (2006). La universidad conectada. Perspectivas del impacto de internet en la educación superior. Málaga: Aljibe.

Piwowar, H., \& Priem, J. (2013). The Power of Altmetrics on a CV. Bulletin of the American Society for Information Science and Technology, 39(4), 10-13. DOI:10.1002/bult.2013.1720390405

Roig, R., Mengual-Andrés, S., \& Guerrero, C. S. (2014). Assessment the pedagogical quality of the MOOC. Profesorado, 18(1), 27-41.

Salager-Meyer, F. (2008). Scientific publishing in developing countries: Challenges for the future. Journal of English for Academic Purposes, 7(2), 121-132. DOI:10.1016/j.jeap.2008.03.009

Sangrà, A. (2002, January 1). A New Learning Model for the Information and Knowledge Society: The Case of the Universitat Oberta de Catalunya (UOC), Spain. The International Review of Research in Open and Distance Learning, 2(2). Retrieved from http://www.irrodl.org/index.php/irrodl/articl e/view/55/114

Schekman, R., Watt, F. M., \& Weigel, D. (2013). A year in the life of eLife. eLife, 2013(2), eLife 2013;2012:e01516. DOI: $\underline{10.7554 / \mathrm{eLife} .01516}$

Schekman, R., Watt, F. M., \& Weigel, D. (2013). eLife and early career researchers. eLife, 2013(2), eLife 2013;2012:e01633. DOI: $10.7554 /$ eLife.01633

Thelwall, M., Haustein, S., Lariviere, V., \& Sugimoto, C. R. (2013). Do Altmetrics Work? Twitter and Ten Other Social Web Services. Plos One, 8(5), 7. DOI: 10.1371 /journal.pone.0064841

Thomson Reuters. (2014). The Research and Innovation Performance of the G20.

Toffler, A. (1985). The Adaptive Corporation. New York: McGraw Hill.

Torres, D., Cabezas, A., \& Jimenez, E. (2013). Altmetrics: New Indicators for Scientific Communication in Web 2.0. Comunicar(41), 53-60. DOI: 10.3916/c412013-05

Walsh, J. P., \& Huang, H. (2014). Local context, academic entrepreneurship and open science: Publication secrecy and commercial activity among Japanese and US scientists. Research Policy, 43(2), 245-260. DOI: $10.1016 /$ j.respol.2013.10.003

Weller, M. (2012). Digital Scholarship and the Tenure Process as an Indicator of Change in Universities. RUSC. Universities and Knowledge Society Journal, 9(2), 167-182, DOI: $\underline{10.7238 / \text { rusc.v9i2.1398 }}$ 


\section{ARTICLE RECORD / FICHA DEL ARTÍCULO}

\begin{tabular}{|c|c|}
\hline $\begin{array}{l}\text { Reference / } \\
\text { Referencia }\end{array}$ & $\begin{array}{l}\text { Duart, Josep M. \& Mengual-Andrés, Santiago (2014). Impacto de la Sociedad del Conocimiento en la } \\
\text { universidad y en la comunicación científica. RELIEVE, v. 20(2), art. M4. DOI: } 10.7203 / \text { relieve.20.2.4343 }\end{array}$ \\
\hline Title / Título & $\begin{array}{l}\text { Impacto de la Sociedad del Conocimiento en la universidad y en la comunicación científica. [Impact of the } \\
\text { Knowledge Society in the University and in Scientific Communication]. }\end{array}$ \\
\hline $\begin{array}{l}\text { Authors / } \\
\text { Autores }\end{array}$ & Duart, Josep M. \& Mengual-Andrés, Santiago \\
\hline Review / Revista & RELIEVE (Revista ELectrónica de Investigación y EValuación Educativa), v. 20 n. 2 \\
\hline ISSN & $1134-4032$ \\
\hline $\begin{array}{l}\text { Publication date / } \\
\text { Fecha de } \\
\text { publicación }\end{array}$ & $\begin{array}{l}\text { Reception Date: } 2014 \text { November 1; Approval Date: } 2014 \text { November 20. Publication Date: } 2014 \\
\text { November } 21\end{array}$ \\
\hline $\begin{array}{l}\text { Abstract / } \\
\text { Resumen }\end{array}$ & 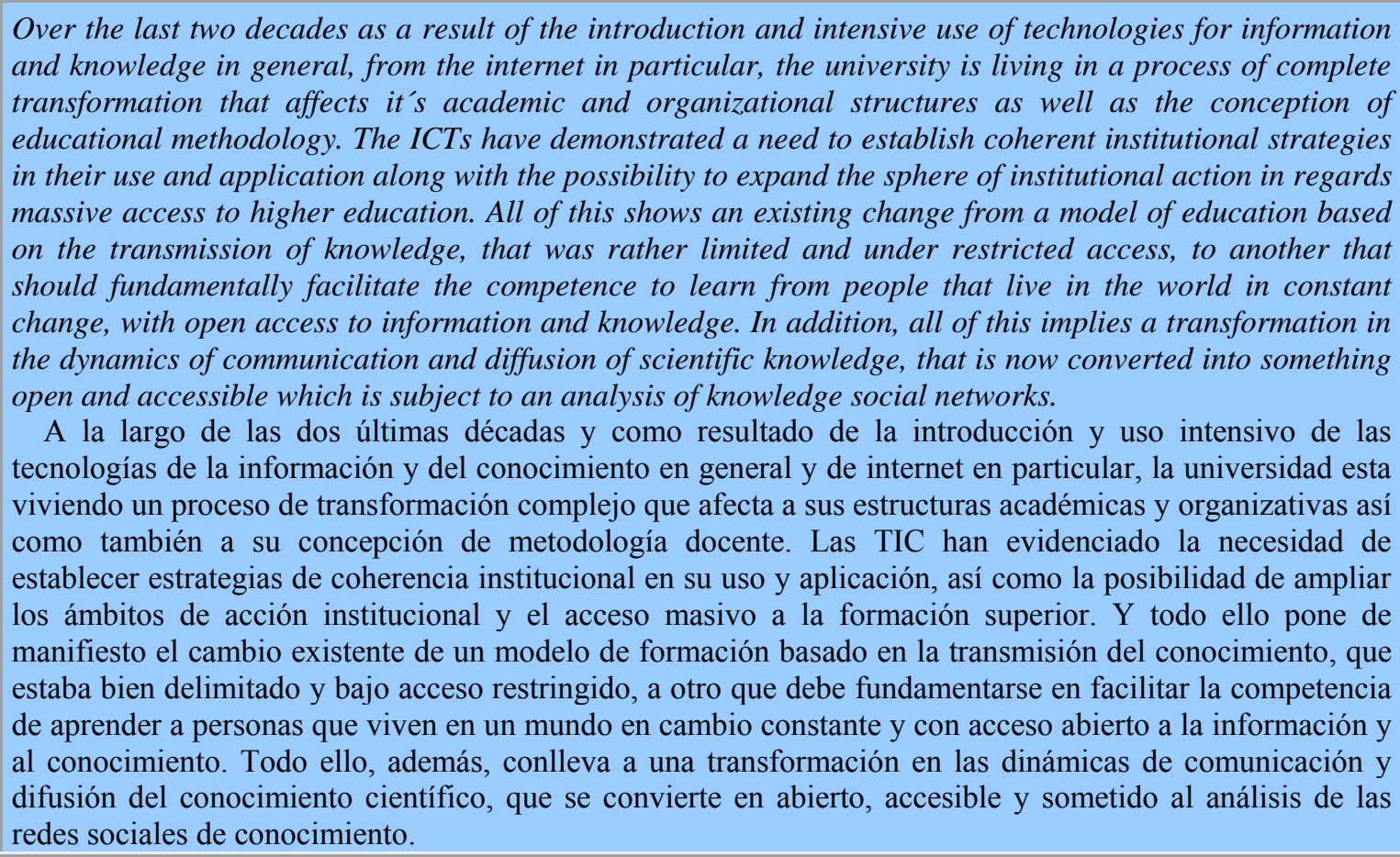 \\
\hline $\begin{array}{l}\text { Keywords / } \\
\text { Descriptores }\end{array}$ & $\begin{array}{l}\text { Higher Education, Knowledge Society, e-learning, Open Acces, Open Science, almetrics. } \\
\text { Educación superior, sociedad del conocimiento, e-learning, acceso abierto, ciencia abierta, altmetric. }\end{array}$ \\
\hline $\begin{array}{l}\text { Institution / } \\
\text { Institución }\end{array}$ & $\begin{array}{l}\text { Autor1: Universitat Oberta de Catalunya (España) } \\
\text { Autor 2: Universidad de Valencia (España) }\end{array}$ \\
\hline $\begin{array}{l}\text { Publication site / } \\
\text { Dirección }\end{array}$ & http://www.uv.es/RELIEVE \\
\hline $\begin{array}{l}\text { Language / } \\
\text { Idioma }\end{array}$ & Español \& English version (Title, abstract and keywords in English \& Spanish) \\
\hline
\end{tabular}

\section{RELIEVE}

\section{Revista ELectrónica de Investigación y EValuación Educativa E-Journal of Educational Research, Assessment and Evaluation}

[ISSN: 1134-4032]

(C) Copyright, RELIEVE. Reproduction and distribution of this articles it is authorized if the content is no modified and their origin is indicated (RELIEVE Journal, volume, number and electronic address of the document).

(C) Copyright, RELIEVE. Se autoriza la reproducción y distribución de este artículo siempre que no se modifique el contenido y se indique su origen (RELIEVE, volumen, número y dirección electrónica del documento). 


\section{ABOUT THE AUTHORS / SOBRE LOS AUTORES}

Duart, Josep M. (jduart@uoc.edu) Doctor en Pedagogía y MBA (Master in Business Administration) por ESADE Business School. Es el autor de contacto para este artículo. Es profesor de la UOC (Universitat Oberta de Catalunya). Es director de RUSC - Revista de Universidad y Sociedad del Conocimiento. Su dirección postal es: Roc Boronat, 117 - Planta 6. 08018 Barcelona (España). Buscar otros artículos de este autor en Google Académico / Find other articles by this author in Scholar Google Google

Mengual-Andrés, Santiago (santiago.mengual@uv.es). Licenciado y Doctor en Psicopedagogía. Profesor del Departamento de Educación Comparada e Historia de la Educación de la Universidad de Valencia (España). Es editor ejecutivo de la revista $\underline{\mathrm{New}}$ Approaches in Educational Research. Su dirección postal es: Avda. Blasco Ibáñez, 30. 46010Valencia (España). Buscar otros artículos de este autor en Google Académico / Find other articles by this author in Scholar Google Google 